\title{
2020 The detection of myocardial scar by ceMRI in patients with TnI positive chest pain and minimal angiographic coronary artery disease
}

\author{
Thomas N Martin*, Bjoern Groenning, Tracey Steedman, John Foster, \\ Alex Elliott, Andrew Flapan and Henry Dargie
}

Address: Glasgow University, Glasgow, UK

* Corresponding author

from I Ith Annual SCMR Scientific Sessions

Los Angeles, CA, USA. I-3 February 2008

Published: 22 October 2008

Journal of Cardiovascular Magnetic Resonance 2008, I0(SuppI I):A289 doi:I0.I I86/I532-429X-I0-SI-A289

This abstract is available from: http://jcmr-online.com/content/I0/SI/A289

(C) 2008 Martin et al; licensee BioMed Central Ltd.

\section{Introduction}

International guidelines recommend early coronary angiography (CA) and revascularization in all patients diagnosed with acute myocardial infarction (AMI). Normal CA does not exclude AMI but makes the final diagnosis less accurate. Contrast enhanced magnetic resonance imaging (ceMRI) is able to both identify myocardial damage and describe its pattern.

\section{Purpose}

To investigate the presence and pattern of delayed hyperenhancement (DE) in troponin positive $(\mathrm{TnI}+)$ patients with normal CA.

\section{Methods}

Eighty consecutive patients with first presentation of TnI+ chest pain underwent ceMRI at a mean (SD) of 64 (23) hours from chest pain on a Siemens Sonata $1.5 \mathrm{~T}$ system using a phased array chest coil during breath-holds. Cinematographic images were acquired using a steady state free precession sequence. CeMRI was performed $>15$ minutes after peripheral injection of $0.2 \mathrm{mmol} / \mathrm{kg}$ gadolinium-DTPA using a segmented gradient-echo inversionrecovery sequence for DE. Scans were assessed by 2 experienced observers and DE was planimetered manually. CA was performed at the discretion of the Cardiologist in charge of patients care, minimal disease is defined as < $25 \%$ maximal stenosis. Patients were divided by pattern of DE into AMI (subendocardial) and cardiomyopathy (subendocardial sparing).

\section{Results}

Eight (10\%) chest pain, TnI+ patients, mean (SD) 46 (12) years, had a normal CA and seven had evidence of DE. Four ( 3 male) had an ischaemic distribution of DE ( 2 inferior, 1 lateral and 1 anterior): median (IQR) TnI, 21.9 (11.3-34.2) ng/ml; DE, 8.1 (2.5-21) g; and minimal disease at CA $(n=3)$. Three ( 2 male) had subendocardial sparing pattern of $\mathrm{DE}$ and were diagnosed with acute myocarditis: median (IQR) TnI, $29.3(14.8-50) \mathrm{ng} / \mathrm{ml}$; $\mathrm{DE}, 36.5$ (21.3-55.6) g; all had normal coronaries at CA. One patient was negative for DE and had a TnI of $4.4 \mathrm{ng} /$ $\mathrm{ml}$; a final diagnosis of Takotsubo cardiomyopathy was made by characteristic cinematographic images. This patient had minimal disease at CA. Only one patient out of eight (12.5\%) had MACE (stroke) recorded at 3 years and he was from the AMI group.

\section{Conclusion}

Ten percent of troponin positive patients in this population had normal coronary arteries. CeMRI has added to the diagnostic accuracy by confirming ischaemic damage in $50 \%$ and diagnosing cardiomyopathy in the other $50 \%$. 